\title{
Phase Space Density Matrix for Emotion Recognition
}

\author{
Mona M. Elamir \\ Biomedical Department \\ Faculty of Engineering \\ Helwan University
}

\author{
Walid Al-Atabany \\ Biomedical Department \\ Faculty of Engineering \\ Helwan University
}

\author{
Mohamed A. Aldosouky \\ Biomedical Department \\ Faculty of Engineering \\ Helwan University
}

\begin{abstract}
Emotion detection of human from physiological signals is one of the active research areas on developing intelligent humanmachine interface systems. Emotions can be expressed either verbally through emotional vocabulary, or by non-verbal such as intonation of voice, facial expressions, gestures and physiological signals. This paper aims to recognize human emotions from electroencephalogram (EEG) signals based on studying the nonlinear behavior of brain signals. Phase space density matrix has been generated from the reconstructed EEG phase space then some features have been extracted using gray level co-occurrence matrix (GLCM) method. Oneway ANOVA test has been used to select the most significant features contributing to emotion classification. Three supervised classifiers (KNN, SVM, and CART classifier) have been used in this study to classify emotions into three cases along the two-basic emotional dimensions. Results show promising preliminary results with average accuracy $95.8 \%$ for arousal dimension and $93.9 \%$ for valence dimension that confirms the robustness of the proposed approach as practical classifier tool for emotion recognition.
\end{abstract}

\section{Keywords}

Emotion recognition, density matrix, phase space, DEAP dataset.

\section{INTRODUCTION}

Affective computing is studying and developing systems and devices that can recognize, interpret, process, and simulate human effects. Emotion detection is one branch of affective computing systems which aims to find out the neural circuits and brain mechanisms of emotion processing. Emotions are a complex state of feeling causing physical and psychological changes that influence thoughts, behaviors, and decision making. They play an important role in many applications such as human-computer interaction applications, considered as an essential feature of human mental health and understanding how we think and behave that has an impact in many applications like security and airplane [1].

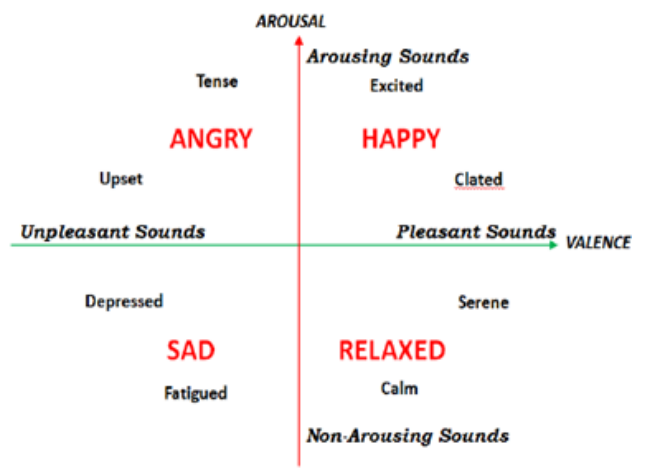

Fig1: shows the two basic dimensions of emotions (arousal-valance)
Emotions can be classified according to many bases; the most common base is classifying emotions into two basic dimensions: (arousal - valance); valence represents the degree of attraction or aversion that individual feels toward a specific object or event. It ranges from negative to positive (happy to sad). However, arousal represents the psychological state that being awake or reactive to stimuli, ranging from passive to active (Excited to sleepy) as seen in Fig1.

To elicit human emotion, an external stimulus has been used which might include: reflexed movements, lights, and colors, set of actions, images (international affective picture system (IAPS) [2] and sounds (international affective digital system (IADS) [3]. Changes in emotional states reflect a response to facial expression, voice tone, handwriting and cause change in physiological signal and gestural modifications. These physiological signals include: (ECG, EEG, EMG, GSR, and temperature) but, EEG is more reliable than others because it records the inner cortical electrical activity of the brain from the scalp.

According to the frontal brain asymmetry concept, the experience of negative emotions is related to an increased right frontal and prefrontal hemisphere activity, whereas positive emotions evoke an enhanced left-hemisphere activity [4]. For that, in case of classifying emotions into happy, sad and neutral, the selected EEG channels should be located on both brain sides. So, two-channels have been selected from the left-hand side, and two on the right-hand side and two on the baseline. Fig2 shows the 10-20 system for EEG channels and the selected channels (Fp1-Fp2-Cz-Fz-F3-F4).

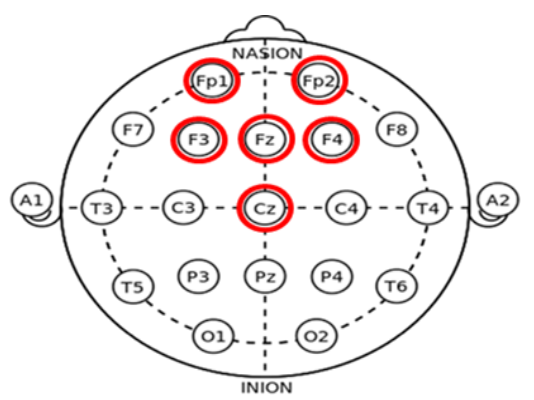

Fig2: EEG channels with selected channels.

There are several studies that handled emotion detection from EEG signals. Some of them have used the available database, others generated their own database. Comparing between these studies is a difficult process because it depends on: physiological signals, the external stimulus, the extracted features, and the used classifiers. Some of these studies are Y.liu and O. Sourina [5] who have compared in their study between the results from two experiments using two different stimuli (pictures from IAPS, and sounds from IADS), their results from DEAP analysis have achieved higher accuracy rate $90.35 \%$ than the other two experiments. Priyanka A. Wandile [6] has achieved an average accuracy $75 \%$ when 
using wavelet transforms to classify EEG signals into four emotions; valence, arousal, dominance, and liking. H. Atasoy and others [7] have used the DEAP database to classify EEG signals into valence, arousal and dominance using some statistical features, entropies and fractal dimension with an average accuracy of $67 \%$ for the valance, $60 \%$ for arousal and $57.58 \%$ for dominance.

Other studies generated their own database such as T. Rached and A. Perkusich [8] have classified EEG signals into positive/excited, positive/calm, negative/excited, and negative/calm using Levenberg-Marquardt, Bayesian regularization, and resilient propagation algorithms. Y. Mohamad [9] has used SVM to classify EEG signal into positive, neutral, and negative by analyzing the signal in both time and frequency domain. S. Lokannavar [10] has achieved 89\% from applying SVM on EEG signal to classify it into four emotional states happy, relax, sad and fearful. P. Petrantonakis and L. Hadjileontiadis have proposed an approach in which only three channels (Fp1, Fp2, and F3/F4) have been used to classify EEG signal into six different states in the valence/arousal space. They have achieved maximum accuracy of $94.40 \%$ [11].

In this paper, a nonlinear analysis technique for emotions recognition from EEG signals has been used to classify EEG signals into three classes in both emotional dimension in which valence dimension has been classified into happy, sad, and neutral and arousal dimension has been classified into excited, calm, and sleepy.

\section{METHODOLOGY}

This paper aims to design an automatic emotion recognition system based on nonlinear analysis for EEG signals using phase space density matrix. Such system classifies EEG signals into two basic dimensions; Valence (happy-neutral-sad) and arousal (Excited-calm-sleepy). . So, we have focused on our analysis of both Beta and Gamma band.

shows the block diagram of the designed system.

EEG signals contain five frequency bands: Alpha, Beta, Gamma, Delta, and Theta. Choosing which band from these bands that related to emotions is a critical point that many researchers are still studying it. In a study for W.Zheng and Bao-Liang Lu [12] who have investigated which frequency band related to emotion, they have found that Gamma and Beta band are the frequency band that related more to emotions than other bands. So, we have focused on our analysis of both Beta and Gamma band.

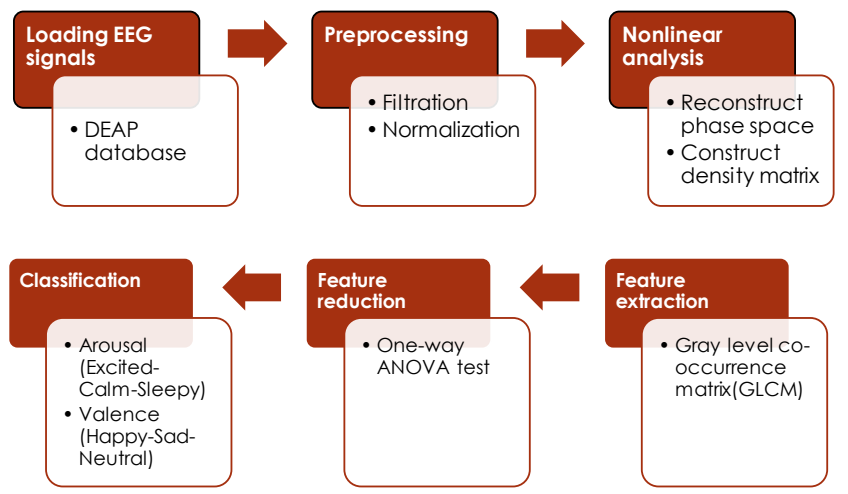

\subsection{DEAP Database}

DEAP dataset (Database for Emotion Analysis using EEG, physiological and video signal) [13] is a multimodal dataset for analyzing human emotions. It includes EEG signal and other peripheral physiological signals (EMG-EOG-GSRtemperature-respiration belt) for 32 participants through watching 40 one-minute musical videos. Participants rated their emotions after each video in terms of continuous values from 1 to 9 along five levels; arousal, valence, like/ dislike, dominance, and familiarity. These dimensions have been defined as the following:

- Arousal refers to how much human feels active (sleepy $=$ low arousal and active $=$ high arousal $)$ toward stimulus.

- Valence refers to how much human feels happy (high valence) or sad (low valence).

- Dominance refers to how much human feels that its emotions dominate him.

- Liking refers only to if the participant liked the video or disliked it.

- Familiarity refers to how much the participant was familiar with the video.

In this paper, EEG signals have been classified into two basic dimensions: valence and arousal. The former dimension classifies signal into 3 groups; sad (taking grades between1-3), neutral (grades between 4- 6) and happy (with grades between 7- 9). However, the later dimension classifies signal into sleepy (from 1-3), clam (from 4- 6) and excited (from 7-9).

\subsection{Preprocessing steps:}

A five order Butterworth band-pass filter has been used for removing noise and selecting the desired band with cutoff frequency band (13:30) $\mathrm{Hz}$ for Beta band and (30-75) $\mathrm{Hz}$ for Gamma band. choosing Butter worth filter is due to its flat response and its simplicity in calculations [14].

\subsection{Phase density matrix}

The nonlinear dynamical system is the system which its behavior changes with time. The human brain is like a complex nonlinear system showing complicated properties as its dynamics changes with time and it exhibits unpredictable (chaotic) behavior. The first step in analyzing dynamical system analysis is reconstructing its phase space using the time delay embedding theorem (Taken's theorem) [15] which calculated from:

$$
\begin{array}{r}
x(i)=y(i)+y(i+\tau)+\cdots+y(i \\
+\tau(m+1))
\end{array}
$$

Where $y(i)$ is the EEG signal, $(m)$ is the embedding dimension and $(\tau)$ is the delay time. The delay time is calculated from the first minimum of the average mutual information function [16] which has been calculated here to be 7 . The embedding dimension is calculated from false nearest neighbors (FNN) method [17] which has been calculated here to be 21 in this work.

The phase space matrix is then generated from the EEG signal $\mathrm{x}(\mathrm{m})$ and its delayed version $\mathrm{x}(\mathrm{m}+\mathrm{t})$ : 


$$
\left[\begin{array}{cccc}
x(1) & x(2) & \ldots & x(m) \\
& \ldots & \\
x(1+t) & x(2+t) & \ldots & x(m+t)
\end{array}\right]
$$
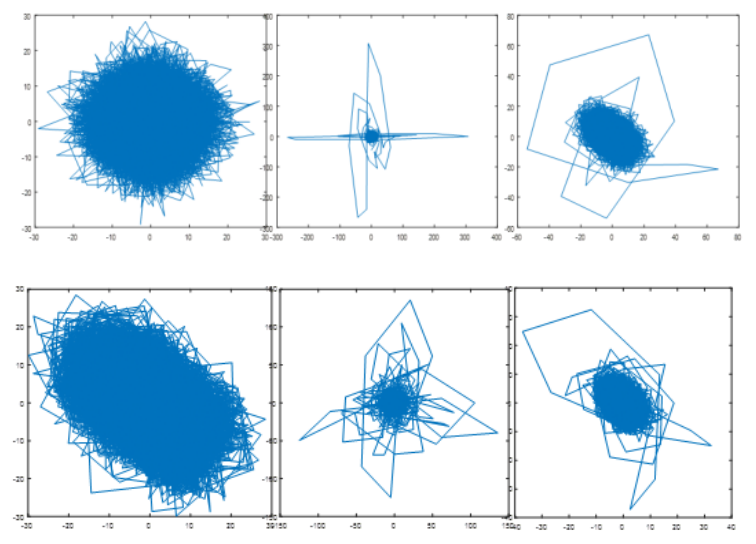

Fig4: (a) shows the Phase space for the three states of valence dimensions; Happy, Sad and neutral, from left to right respectively. (b) Phase space for the three states of Arousal dimensions; excited, sleepy and calm, from left to right respectively.

The two-dimension phase space plot has been obtained from plotting the second column against the first one 1 of the reconstructed phase space matrix. Error! Reference source not found. shows the phase space trajectory for the three classes of both valence and arousal dimensions. Now after generating the phase space density matrix, the phase space plot area has been divided into $20 \times 20$ equal sizes squares. A phase space density matrix $\mathrm{C}$ has been obtained with its elements $C(i, j)$ from the mean of each grid:

$$
=\left[\begin{array}{lll}
\sum_{i g, j g}^{n} \mu(i=1, j=1) & \ldots & \sum_{i g, j g}^{n} \mu(i=1, j=20) \\
\sum_{i g, j g}^{n} \mu(i=2, j=1) & \ldots & \sum_{i g, j g}^{n} \mu(i=2, j=20) \\
& \cdot \\
\sum_{i g, j g}^{n} \mu(i=20, j=1) & \ldots & \sum_{i g, j g}^{n} \mu(i=20, j=20)
\end{array}\right]
$$

It is observed that the distributions of phase space points for the different emotion are clearly different in the phase space density matrix plots as shown in Fig.

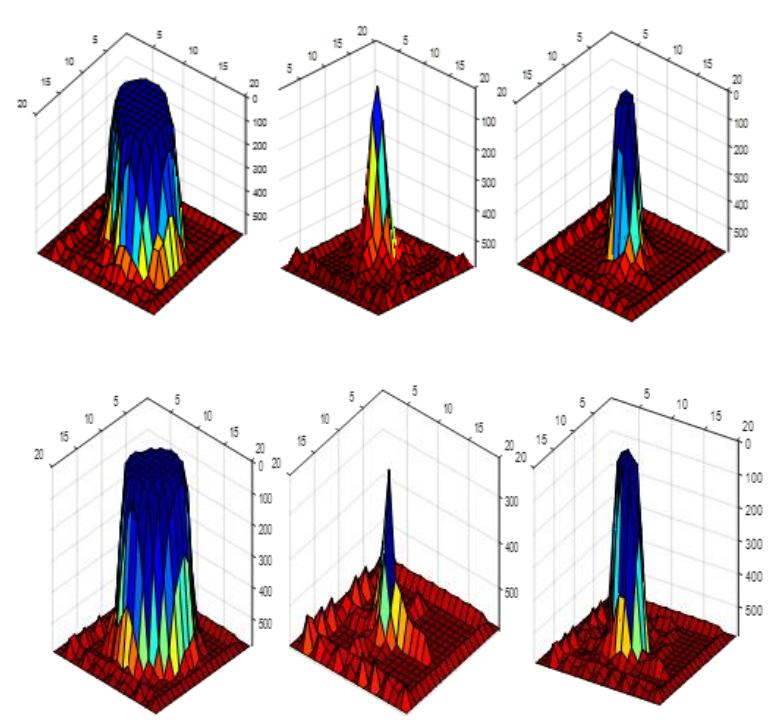

Fig5: (a) Density matrix reconstructed from the twodimensional phase spaces for the three cases of arousal dimension, from left to right(Excited, Calm and sleepy). (b) Density matrix reconstructed from the twodimensional phase spaces for the three cases of Valence dimension, from left to right(Happy-neutral - sad).

The reconstructed phase space density matrix contains 400 elements in the newly generated density matrix that are too much to be used as a feature that proposed to the classification process. So, the gray level co-occurrence matrix (GLCM) technique [18] has been applied to extract features from the density plot, GLCM is a statistical method for examining the texture discrimination. Each element in the normalized GLCM is the joint probability occurrence of pixel pairs with a defined spatial relationship having a gray level value $i$ and $j$ in the image. GLCM has four major properties that summarized below:

A. Contrast: measure the intensity contrast between a pixel and its neighbor over the whole image:

$$
\text { contrast }=\sum_{i, j}(i-j)^{2} P(i, j)
$$

$\mathrm{P}$ is the probability of the two pixels

b. Correlation: measure how pixel correlated with its neighbor over the whole image:

$$
\operatorname{corr}=\sum_{i, j} \frac{(i-\mu i)(j-\mu j)}{\sigma_{i} \sigma_{j}}
$$

$\mu$ is mean and $\sigma$ is the variance. Correlation is either 1 or -1 for a perfectly positively or negatively correlated image.

c. Energy: Returns the sum of squared elements in the GLCM:

$$
\text { Energy }=\sum_{i, j} P(i, j)^{2}
$$

Energy range [0:1], Energy $=1$ for a constant image. This property is also known as uniformity, uniformity of energy, and angular second moment.

d. Homogeneity: Returns a value that measures the closeness of the distribution of elements in the GLCM to 
the GLCM diagonal. (Homogeneity is 1 for a diagonal GLCM).

$$
\text { Heто }=\sum_{i, j} \frac{P(i, j)}{1+(i-j)}
$$

\section{RESULT AND DISCUSSION}

As mentioned earlier, DEAP Database has been used in our proposed automatic emotion recognition system. Such dataset consists of more than one-thousand signals recorded from 32 participants who have been asked to watch 40 videos containing the three emotional cases for each dimension. The two-dimensional phase space trajectory of the signal has been reconstructed using the delay time embedding method. Then the phase space plots of the signals have been divided by a grid of 20x20 squares and the numbers of points $C(i, j)$ within each square have been computed to form the phase space density matrix $C$. Four features have been extracted from the GLCM matrix from both Beta and Gamma band of the six EEG channels (Fp1, Fp2, Cz, Fz, F3, and F4) to form the features vectors. There are several approaches to assess the significance of the extracted features such as Fisher projection, ANOVA, and sequential feature selection.

In this paper, one-way analysis of variance (ANOVA) [19] has been chosen to test the significances of the extracted features. Error! Reference source not found. shows the p-value for all features that have been obtained from applying ANOVA test.

Table 1. the resultant p-values from applying one-way ANOVA test on our extracted features from Gamma band.

\begin{tabular}{|l|l|l|l|l|l|l|}
\hline \multirow{2}{*}{} & \multicolumn{6}{|l|}{ p-values for ANOVA test } \\
\cline { 2 - 7 } & Fp1 & $\mathbf{F p 2}$ & $\mathbf{C z}$ & $\mathbf{F z}$ & $\mathbf{F 3}$ & $\mathbf{F 4}$ \\
\hline Contrast & $\mathbf{0 . 0 1 2}$ & $\mathbf{0 . 0 2 9 4}$ & 0.6055 & $\mathbf{0 . 0 1 2 3}$ & $\mathbf{0 . 0 1 9}$ & $\mathbf{0 . 0 0 2}$ \\
\hline Correlation & $\mathbf{0 . 0 1 8}$ & $\mathbf{0 . 2 0 8 1}$ & $\mathbf{3 . 4} * 10-5$ & $\mathbf{0 . 0 1 8 7}$ & 0.48 & $\mathbf{0 . 0 0 6}$ \\
\hline Energy & $\mathbf{0 . 0 1 1}$ & $\mathbf{0 . 0 2 0 9}$ & 0.703 & $\mathbf{0 . 0 1 1 2}$ & $\mathbf{0 . 0 1 3}$ & $\mathbf{0 . 0 0 0 1}$ \\
\hline $\begin{array}{l}\text { Homogenit } \\
\text { y }\end{array}$ & $\mathbf{0 . 0 4 4}$ & 0.0943 & 0.2628 & $\mathbf{0 . 0 4 4 1}$ & 0.117 & $\mathbf{0 . 0 2}$ \\
\hline
\end{tabular}

Table 2. Resultant p-values from applying one-way ANOVA test on our extracted features from the Beta band.

\begin{tabular}{|l|l|l|l|l|l|l|}
\hline \multirow{2}{*}{} & \multicolumn{6}{|l|}{ p-values for ANOVA test } \\
\cline { 2 - 7 } & $\mathbf{F p 1}$ & $\mathbf{F p 2}$ & $\mathbf{C z}$ & $\mathbf{F z}$ & $\mathbf{F 3}$ & $\mathbf{F 4}$ \\
\hline Contrast & $\mathbf{0 . 0 2 3}$ & $\mathbf{0 . 0 3 1}$ & 0.598 & $\mathbf{0 . 0 3 5 1}$ & $\mathbf{0 . 0 2 3}$ & $\mathbf{0 . 0 0 5}$ \\
\hline Correlation & $\mathbf{0 . 0 3 8}$ & 0.312 & $\mathbf{1 . 3} * 10-4$ & $\mathbf{0 . 0 2 0 1}$ & 0.51 & $\mathbf{0 . 0 1 2}$ \\
\hline Energy & 0.068 & $\mathbf{0 . 0 3 2}$ & 0.693 & $\mathbf{0 . 0 0 2}$ & $\mathbf{0 . 0 2 0}$ & $\mathbf{0 . 0 2 3}$ \\
\hline $\begin{array}{l}\text { Homogenit } \\
\text { y }\end{array}$ & $\mathbf{0 . 0 1 5}$ & 0.11 & 0.3012 & 0.0501 & 0.201 & 0.102 \\
\hline
\end{tabular}

Before applying ANOVA test, the feature vector size has contained 24 features for six EEG channels at each frequency band, this number will consume large classification time, but after ANOVA test it has become only 18 featured from Gamma band and only 14 features for the Beta band which had the significant $p$ values. Three statistical classifiers have been used in this paper; support vector machine (SVM) [20], k-nearest neighbor (KNN) [21] and classification and regression tree (CART) [22] classifiers.
To calculate accuracies for the three classifiers; sensitivity and specificity [23] for each classifier have been calculated from the following formula:

$$
\begin{gathered}
\text { Senstivity }=\frac{T P}{T P+F N} \\
\text { Specificity }=\frac{T N}{T N+F P} \\
\text { Accuracy }=\frac{T N+T P}{T P+T N+F P+F N}
\end{gathered}
$$

Such:

TP: True positive; number of correct observations which have no disease.

TN: True negative; number of correct observations which have disease.

FP: False positive; number of correct observations which

have no disease where they have a disease.

FN: False negative; number of correct observations which

have a disease where they haven't a disease.

Table 3. Sensitivity and specificity for the three classifiers along the two dimensions at Gamma band

\begin{tabular}{|l|l|l|l|l|l|l|}
\hline \multirow{2}{*}{} & \multicolumn{2}{|l|}{ Arousal } & \multicolumn{2}{l|}{ Valence } \\
\cline { 2 - 7 } & SVM & KNN & RT & SVM & KNN & RT \\
\hline Senstivity\% & 95.8 & 93.5 & 91 & 92 & 90.5 & 96.9 \\
\hline Specificity\% & 95.8 & 94.9 & 94 & 96.4 & 90.5 & 95 \\
\hline Accuracy\% & $\mathbf{9 5 . 8}$ & 94.2 & 92.5 & 94.2 & 90.25 & $\mathbf{9 5 . 8}$ \\
\hline
\end{tabular}

Table 4. Sensitivity and specificity for the three classifiers along the two dimensions at the Beta band

\begin{tabular}{|l|l|l|l|l|l|l|}
\hline \multirow{2}{*}{} & \multicolumn{3}{|l|}{ Arousal } & \multicolumn{3}{|l|}{ Valence } \\
\cline { 2 - 7 } & SVM & KNN & RT & SVM & KNN & RT \\
\hline Senstivity\% & 90 & 94 & 93.4 & 92.4 & 91.4 & 94 \\
\hline Specificity\% & 93 & 92 & 93.1 & 88.1 & 91.9 & 91.2 \\
\hline Accuracy\% & 91.5 & 93 & $\mathbf{9 3 . 2}$ & 90.25 & 91.65 & $\mathbf{9 2 . 6}$ \\
\hline
\end{tabular}

Error! Reference source not found. and Table 4 show the average accuracies achieved by phase space density matrix technique from EEG signals in both Beta and Gamma bands; it is obvious that Gamma band records higher accuracy rate than the Beta band which means that EEG signal in Gamma band is related more with the human emotion variation.

To evaluate our work, our proposed technique has been compared with other studies that used the same dataset as shown in Error! Reference source not found.

Table 5. The comparing table between density matrix technique and other techniques which use the same database.

\begin{tabular}{|ll|l|l|}
\hline \multicolumn{2}{|c|}{ Technique } & $\begin{array}{l}\text { No. of } \\
\text { group }\end{array}$ & Accuracy \\
\hline Fast & Fourier Transform & 4 & $82 \%$ \\
\hline
\end{tabular}




\begin{tabular}{|l|l|l|}
\hline (FFT) [24] & & \\
\hline $\begin{array}{l}\text { Auto Regression, FFT, } \\
\text { fractal dimension [10] }\end{array}$ & 4 & $89 \%$ \\
\hline $\begin{array}{l}\text { HOC and statistical } \\
\text { analysis [5] }\end{array}$ & 8 & $90.35 \%$ \\
\hline Differential entropy [12] & 3 & $\begin{array}{l}\text { Max achieved } \\
\text { accuracy 86.65\% }\end{array}$ \\
\hline $\begin{array}{l}\text { Phase space density } \\
\text { matrix (this study) }\end{array}$ & 6 & $\begin{array}{l}95.8 \% \text { for both } \\
\text { arousal and valence } \\
\text { dimensions at } \\
\text { Gamma band }\end{array}$ \\
\hline
\end{tabular}

\section{CONCLUSION}

In this paper, an automatic emotion recognition system has been presented based on nonlinear analysis for EEG signal. It has shown that the features extracted from the reconstructed phase space density of the EEG signals were promising in recognizing and classifying human emotion. Using nonlinear analysis can help in enhancing results as it helps in extracting more information about EEG signals.

Results show that emotion recognition from the nonlinear analysis is a robustness technique that can be applied in different applications. This promising result has encouraged us for applying more physiological signals in emotion recognition. Gamma band has achieved higher accuracy rate than a Beta band that means, it is the most frequency band related to emotion.

\section{Conflict of Interest}

The authors declare that there is no conflict of interest regarding the publication of this paper.

\section{REFERENCES}

[1] Lisetti, CL," Affective computing", Springer, 1998.

[2] (http://csea.phhp.ufl.edu/Media.html\#topmedia).

[3] (http://csea.phhp.ufl.edu/media.html\#midmedia).

[4] P. C. Petrantonakis and L. J. Hadjileontiadis, "A novel emotion elicitation index using frontal brain asymmetry for enhanced EEG-based emotion recognition," IEEE Transactions on information technology in biomedicine, vol. 15 , no. 5 , pp. 737-746, 2011.

[5] Y. Liu and O. Sourina, "EEG databases for emotion recognition," in Cyberworlds $(C W), 2013$ International Conference on, 2013, pp. 302-309: IEEE.

[6] P. A. Wandile, N. Bawane, and M. P. Hajare, "Emotion Detection from Brain and Audio Signal."

[7] H. Atasoy, S. Yıldırım, E. Yıldırım, and Y. Kutlu, "EEG Sinyallerinden Fraktal Boyut Ve Dalgacık Dönüşümü Kullanılarak Duygu Tanıma Emotion Recognition from EEG Signals Using Fractal Dimension And Wavelet Transform."

[8] T. S. Rached and A. Perkusich, "Emotion recognition based on brain-computer interface systems," in Braincomputer interface systems-Recent progress and future prospects: InTech, 2013.

[9] Y. Mohamad et al., "Detection and utilization of emotional state for disabled users," in International Conference on Computers for Handicapped Persons,
2014, pp. 248-255: Springer.

[10] S. Lokannavar, P. Lahane, A. Gangurde, and P. Chidre, "Emotion recognition using EEG signals," Emotion, vol. 4, no. 5, pp. 54-56, 2015.

[11] P. C. Petrantonakis and L. J. Hadjileontiadis, "Emotion recognition from EEG using higher order crossings," IEEE Transactions on Information Technology in Biomedicine, vol. 14, no. 2, pp. 186-197, 2010.

[12] W.-L. Zheng and B.-L. Lu, "Investigating critical frequency bands and channels for EEG-based emotion recognition with deep neural networks," IEEE Transactions on Autonomous Mental Development, vol. 7, no. 3, pp. 162-175, 2015.

[13] S. Koelstra et al., "Deap: A database for emotion analysis; using physiological signals," IEEE Transactions on Affective Computing, vol. 3, no. 1, pp. 18-31, 2012.

[14] I. W. Selesnick and C. S. Burrus, "Generalized digital Butterworth filter design," IEEE Transactions on signal processing, vol. 46, no. 6, pp. 1688-1694, 1998.

[15] J. Stark, D. Broomhead, M. Davies, and J. Huke, "Takens embedding theorems for forced and stochastic systems," Nonlinear Analysis: Theory, Methods \& Applications, vol. 30, no. 8, pp. 5303-5314, 1997.

[16] A. M. Fraser and H. L. Swinney, "Independent coordinates for strange attractors from mutual information," Physical review A, vol. 33, no. 2, p. 1134, 1986.

[17] H. D. Abarbanel and M. B. Kennel, "Local false nearest neighbors and dynamical dimensions from observed chaotic data," Physical Review E, vol. 47, no. 5, p. 3057, 1993.

[18] D. A. Clausi, "An analysis of co-occurrence texture statistics as a function of grey level quantization," Canadian Journal of remote sensing, vol. 28, no. 1, pp. 45-62, 2002.

[19] A. Mayers, Introduction to Statistics and SPSS in Psychology. Pearson Higher Ed, 2013.

[20] I. Steinwart and A. Christmann, Support vector machines. Springer Science \& Business Media, 2008.

[21] P. Soucy and G. W. Mineau, "A simple KNN algorithm for text categorization," in Data Mining, 2001. ICDM 2001, Proceedings IEEE International Conference on, 2001, pp. 647-648: IEEE.

[22] L. Breiman, Classification and regression trees. Routledge, 2017.

[23] D. G. Altman and J. M. Bland, "Diagnostic tests. 1: Sensitivity and specificity," BMJ: British Medical Journal, vol. 308, no. 6943, p. 1552, 1994.

[24] A. Al-Nafjan, M. Hosny, A. Al-Wabil, and Y. Al-Ohali, "Classification of Human Emotions from Electroencephalogram (EEG) Signal using Deep Neural Network," INTERNATIONAL JOURNAL OF ADVANCED COMPUTER SCIENCE AND APPLICATIONS, vol. 8, no. 9, pp. 419-425, 2017 
International Journal of Computer Applications (0975 - 8887)

Volume 179 - No.49, June 2018

IJCATM : www.ijcaonline.org 